\title{
Combination of ultrasound and ultraviolet-C irradiation on kinetics of color, firmness, weight loss, and total phenolic content changes in tomatoes during storage
}

\begin{abstract}
A kinetic study was conducted to account for the changes occurring in color, firmness, weight loss, and total phenolic content of tomatoes subjected to combined ultrasound technology (40 $\mathrm{kHz}, 13.78 \mathrm{~W} / \mathrm{L})$ and ultraviolet-C radiation $(639.37 \mu \mathrm{w} / \mathrm{cm} 2)$ treatment for $28 \mathrm{~min}$. Combined treatment delayed ripening, increased firmness, weight loss, and induced total phenolic content retention of tomatoes compared to untreated samples. The change in color parameters of hue angle and chroma was described using the fractional conversion and zeroorder models, respectively. Fractional conversion kinetic model was also found suitable for firmness degradation, while the zero-order and first-order models were appropriate for change in weight loss and total phenolic content retention prediction, respectively. Activation energy for measured parameters ranged from 121.29 to $199.70 \mathrm{~kJ} / \mathrm{mol}$ at a temperature range of $33.76-37.12^{\circ} \mathrm{C}$, and showed highest sensitivity to treatment temperature in reduced ranking for firmness degradation, weight loss, total phenolic content retention, hue angle, and chroma.
\end{abstract}

